\title{
Hacia un modelo de servicios en la biblioteca digital*
}

\author{
GeOrgina ARACELITORRES VARgaS \\ CentroUniversitariodeInvestigacionesBibliotecológicas \\ de la UNAM, 04510, Méxi co D.F., Tel: 56-23-03-61 \\ E-Mail:gatv@servidor.unam.mx
}

\begin{abstract}
RESUMEN
Se identifican los servicios que se ofrecen en la biblioteca digital y a partir de ello se analiza si éstos son o no novedosos, tomando como punto de comparación a los servicios tradicionales. El objetivo principal de este estudio es conocer cuál es el modelo de servicios que subyace en la biblioteca digital y sus características, como un primer paso para la propuesta de un modelo de servicios para la biblioteca digital.
\end{abstract}

Palabras Clave:Bibliotecas digitales; Servicios de información; Modelo de servicios.

\section{TOWARDS A MODEL FOR DIGITAL LIBRARY SERVICES GEORGINA ARACELITORRES-VARGAS}

\begin{abstract}
The services offered in a digital library are identified and on that basis an analysis is made to determine whether these are new or not, compared with traditional services. The main aim of the paper is to find out which is the model for services underlying the digital library as well as its characteristics. This a first step towards a proposal for a model of digital library services.

Keywords: Digital Libraries; Information Services; Model of Services.
\end{abstract}

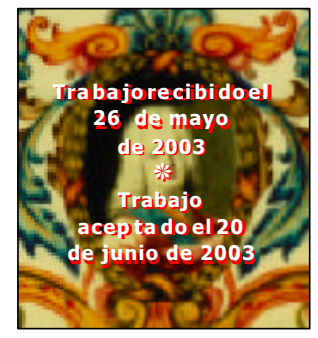

\section{INTRODUCCIÓN}

\begin{abstract}
A partirdeladécada de 1990 surgió un fuer te in te rés por el es tu dio dela bi blio teca

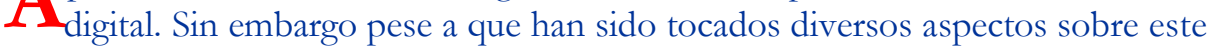
tema, se nota una escasa literatura que analice a fondo lo relativo a los servicios. Cier to que en tex tos y even tos aca dé mi cos so bre el tema se hace una cons tan temención so bre los servicios que de ben ofre cer se en una biblio te ca di gi tal, pero no se detecta ningún trabajo en donde éstos se analicen.
\end{abstract}

\footnotetext{
* Elpresen tees crito de riva de un proyec to de in ves tiga ción apoya do porelConsejo Na cio nal de CienciayTecnología.
} 
Porloge ne rallos trabajos que alu den a los ser vi cios de la biblio te ca digital suelen res trin girse a opi nio nes de ca rác terge ne ral en las que se ase ve ra queés tos re pre sentan un cam bio sus tan cial y que fren te a aquellos que se ofre cen en la bi blio te ca tra dicional,sontotalmentenovedosos.

En con se cuen cia han surgi do te mas de es tu dio que se rela cionan con este su puesto cam bio, en trelos que estáel pa pelquejuegaelbiblio te cólogoantelos nuevos re tos del am bien te di gi tal, el que por ser un tema am plio e incluir pun tos de vis ta di ver sos hasidoabordadoentrabajosparticulares.

Lo mis mo ocurre con otros te mas que de al gu na for mase relacio nan conlos servicios dela bi bliote ca digital, por lo que es ne ce sa rio pre cisarque el pre sen te tra bajo se limitará al análisis de los servicios que se ofre cen en la bi blio te ca di gital, sin atender aspectoscolateralesquerequeriríandeotrosestudios.

Frentealas ase veracionesop timistasype simistasquehaysobreloqueesunabibliote ca digitaldebemos analizardela manera másobjetiva silos servicios deestabibliotecasonononovedosos, en compara ciónconlosqueofrecelabiblio te ca tradicionalye n quémedidaloson. Se trata de conocercuál es el mo delodeserviciosque subyace alabibliotecadigital,esdecir,cuáles sonlosserviciosqueofreceycuálessus propiedades.

Tomando en cuenta que un modelo teórico se considera una aproximación, el presen tees tu dio toma como re fe rencialas pro piedades delosserviciosquese observanenlabibliotecadigitalparaanalizarlosoexplicarlos.

Es frecuen teque un modelo teó rico se formule conbase en una analogía en tre el sistema en cuestión y algún otro sistema conocido que esté gobernado por leyes que se entien den, porloquesilabiblio te ca tra dicional refleja un modelodeserviciosyaconocido, para efec tos de este es tu dio será de gran utilidad es table ceruna ana logía en trelos servicios delabibliotecatradicionalyaquellosqueseobservanenlabibliotecadigital.

Ave riguarcuáles el mo delode servi ciosquese pre senta enlabiblio te cadigital, podría ayu darnos a com pren dersus al can ces y li mi ta cio nes como un pri mer paso para hacerunapropuestasobreunmodelodeserviciosparalabibliotecadigital.

\section{QUÉ ES UNA BIBLIOTECA DIGITAL}

Paracomenzaresnecesariopartirdeunadefinicióndebibliotecadigital.

Conbase en el análisis de diversas de finiciones delabibliotecadigital, ${ }^{1}$ pue de decirsequeidealmenteéstatienelos siguientesrasgos:

* Todasuin formaciónes digital(tex tocompleto,imágenes,y/ocombina ción de diversos medios).

* Tiene conexión en red.

* Cuenta con catálogos públicos en línea.

1 Cfr. Georgina AraceliTorres Vargas.Labibliotecavirtual ¿quéesy quépromete? :UNAM, CUIB, 2000. 
Permite el accesoremoto a los recursos de in formacióndeotras bibliotecas o repositorios.

Ofrece acceso universal a los documentos.

No hay que perderde vis ta que laidea de bi blio te ca digital surge a partirdela apa rición de redes como Internet, pues ha sido pensada para trabajar en medios como éste, por lo que no es raro pen sar en que la bi blio te ca di gi tal des can se so bre una base sistémica.

Por lo tan to con si de ro quela bi blio te ca di gi tal ovir tual se de fine como una red de herramientastec nológicas, contenidosy servicios, que pueden serlocaliza dos endiferenteslatitudesdelmundo.

Elacce so que este tipo de biblio teca pueda ofre cer de pen de rá de sugra do de conectividad con las re des de cóm pu to y dela dis ponibilidad de sus con te ni dos, así como de losprogramas decómputodelosquedis pongaelmercadoenesemomento.

Los con te nidos, a suvez, deben serdis pues tos de ma ne ra digital, conlo que se estable ce una rela ción directa conla publica ción digi tal y conlos facto res que se asocian con ella. ${ }^{2}$ Como seña la García, ${ }^{3}$ los fondos de la biblioteca digitalseráncolecciones dema te riales en forma digital; unos crea dos en forma to digitaly otros conver ti dos a esteformatoapartirdedocumentostextualesimpresos.

Porcon siguien te este tipo de biblio te cas ha dado paso a un fuer te de sa rrollo en la produccióny elusodedocumentos dena turaleza digital, comolos quese encuentran ac tual men te en In ter net. Esto a su vez de man da la bús que da de solu cio nes para emprenderunaorganizaciónyrecuperaciónadecuadadedocumentos digitales.

Generalmente pueden usarse casi todos los textos completos de artículos de revis tas yobras decon sulta, aun que algu nos síre quie ren un pago previopor elservicio. Sinembargolas políticas deIn ternetnoestán comprometidasconla pre servaciónde informa ción, carac terís tica que quizá podráversecon mayorfuerza en una pos te rior fase de de sa rrollo dela bi blio te ca di gital, en la que se cree que ha brá colec cio nes con informacióngeneradasólodeformadigital.

Las ba ses deda tos tam bién cons ti tuyen un elemen to esencial delabiblio te ca di gital, por lo que mu chas veces se con si de ra que al gu nas ba ses de da tos ubica das en sitios web son ejem plos de biblio tecas digitales, locuales completa men te erróneo.Las bases de datos por sí mis mas no son biblio te cas, sino uno de sus com po nen tes. En este sen tido una se rie de ca tálo gos de biblio te cas se es tán ponien doen red para ha cer accesiblelos registrosdesus documentosvíaInternet.

La bi blio te ca digi tal se cons ti tu ye a par tir de la tec no lo gía de que se dis pon ga en el mercado, por lo que esta bi blio te ca es sus cep tible de ad qui rirnuevos ras gos, lo que dependedelcontex to omomentotecnológicoenqueseubique.Ocomoalgunasvecesse

2 Entre las que resaltanlosmecanis mosdecomercialización.

3 Ernes to García Ca ma rero.Labibliotecadigital / Ernes to García Ca ma re ro,Luis Angel García Mele ro.- Madrid : Arcos, 2001. 
afirma, los servicios deestabiblio teca es tán es tre chamente relacio na dos conlas herramientastecnológicasquesurgen. ${ }^{4}$

Es poreso que parateneracce soalas colec cio nes delabibliote ca digital, el usuario re quie re dis po ner de tec no lo gía apro pia da y sa ber acerca de su ma nejo y uso, y tambiénquelos contenidos (tex tos, imágenes, da tos, etcétera) sólopuedenserutilizados atravésdeprogramasespecíficos.

En relación con el acceso, la biblioteca digital puede ser un medio para acceder universal mente a los documentos digitales dispues tos en red, peronopara accedera todo el universo de publi ca cio nes, ya que los im pre sos no for ma rían par te de sus colecciones. Hayqueseñalarqueen reite radasocasiones seconsiderauniversalidad a la identifica ción de publica cionesa tra vés de ca tálogospúblicosenlínea odeca tálogos enWeb, queofrecelabibliotecadigital.

Sin embargo el ras go de universalidad se está rela cio nandocada vez más conlabibliotecahíbrida -ahoratambiéndenominada bibliotecacomplejaomix ta-que comenzóaplantearsedesde1998.

\section{LOS SERVICIOS EN LA BIBLIOTECA TRADICIONAL}

Los servicios son esen ciales para toda biblio te ca, sinellos se ría sóloun al ma cén de libros. A tra vés de los servicios se da el en la ce en trelas co lec cio nes y los usua rios, y se lespermiteaestosúltimosunmejoraccesoalain formación.

Sin em bargopese a su im portancia, el tema de los servicios biblio te ca rios co menzó a ocu parun lugarcen tral a partir del mo vi mien to biblio te ca rio del sigloXIX, momentoen elqueseperfilalaideamodernadebiblioteca.

Elabordaje delos servi cios biblioteca rios como tema dees tu dio po seehoyva rias aris tas, mu chas delas cuales es tán sien do ana liza das porlos es pe cia lis tas. Pese a ello parece ha ber un va cío en lo que res pec ta al aná li sis de los ser vi cios des de su origen, pues es bienco no cido que exis ten diferentes tipos de servicios y sus bon da des, pero no cómo surgen y con qué pro pó si to. En este sen ti do es im por tan te to maren cuen ta lanatu ra leza delos servicios bibliotecarios delabiblio tecatra dicional, loscontextos enlosquesurgenyquécaracterísticasleotorganalabibliotecaquehas tahoyimpera.

En un escritoan te rior ${ }^{5}$ analizabacómolabiblio te ca tradicional fueadquirien doa tra vés del tiem polos servi cios y ca rac te rís ticas quehoy po see. De esta forma se puedendistinguirdiversospropósitosdelosmismos:

Los serviciosbibliotecarios aparecen a par tir de que en la bi blio te ca se tie nela preocupación por elregis trodelos documentosy, sobre todo, a partirdelasis te ma tización delos materiales que con for manlas colec ciones. ${ }^{6}$ El ca tálo go ocu pa unlugar

4 Ibidem.

5 Georgina AraceliTorres Vargas. "Los servicios biblioteca riosy dein forma ción en el con tex to de la biblio te ca tra dicional”, en Investigación bibliotecológica. - Vol. 15, no. 31. - (jul.-dic., 2001). - p. 112.

6 D.J. Foskett. en Information service in libraries. - India : Akashdeep, 1992. - p. 11. 
central en eles tablecimien to delosservicios, comome dioa través delcual seidentificanlosmateriales.

Graciasalasistematización demateriales, surgen servicios comolosde préstamo yconsulta.

Porotra parte, de bi do a que se da una ma yor aper tu ra en el uso de las co lec cio nes de las bi blio te cas, surge la idea del serviciopúblico, en don de la gra tui dad jue ga un papelesencial. ${ }^{7}$ Este mo delo aún per sis te en mu chas delas biblio te cas tra dicionales actuales.

Posteriormenteaparecelacooperación bibliotecaria, quepermi tela apa riciónde los servi cios deinformación, en dondelos ma teriales pue denidentificarseatra vés de bases de da tosy ca tálogos enlínea, ${ }^{8}$ y don de porcon siguien te tales mate rialespueden o no ser par te de la co lec ción de la biblio te ca des de la cual se con sul ta el ca tálogo. Lacooperaciónsurgejuntoconlaposibilidaddecompartirrecursosdocumentales.

Sibienes cier to quela co opera ciónya es ta bapresen te a tra vés del prés ta mo in terbibliotecario, es sólo recientemente cuando se convierte en un servicio sustancial. De este modolabiblio te ca co mien za a darle prio ridad a laiden tifica ción delos do cut mentos, másqueasuposesión física.

Jun to con lo an te rior apa re cen ser vi cios como la diseminación selectiva de la información y laentregadedocumentosenlínea.

Tales son los servicios que actualmente ofrece la biblioteca digital, intentemos ahoradaralgunoselementosconlaintencióndedefinirunmodelodeservicios.

\section{MODELODESERVICIOSENLABIBLIOTECADIGITAL}

Elmo delo de servi cios que aquí se pre sen ta se des pren de del análisis previo efectuadosobrealgunos proyectos debiblio te cas digitales endiversaspartes delmundo. ${ }^{9}$ La identificación de los servicios que ofrecen estas bibliotecas y sus características permitieronestablecerlaanalogía corres pondienteconlos serviciosqueotorgalab ibliotecatradicional.

Enelcontex todelabibliote catra dicionallacoleccióneslomásimportante,razón porla que se sigue ponien do ungran én fa sis en elin cre men to y pro tec ción de ésta. ${ }^{10}$ No obs tante el pa pel de sem pe ña do porla colec ción sigue pre vale cien do den tro del am bien te di gital.La preserva ción dela he ren cia cul tu ral es uno delos ob je tivosque persiguen mu chos de los pro yec tos de biblio te ca digital, comolo ilus tra el pro yec to

7 Bernard J. Hurley. The making of America II testbed project : a digitallibrary service mo del. -Washington, D.C. :The Digital Library Federation, 2000. - p. 13.

8 Su san K. Mar tín.Librarynetworks, 1986-87:librariesinpartnership. - New York : Knowledge In dustry Publica tions, 1986. - p. 12.

9 Como los pro yec tos de la Na tio nal Scien ce Fun da tion, la Bri tish Library, La Biblio te ca Nacional de Francia, y diversos proyectos que se lle van a cabo en Mé xi co.

10 Stan A. Hannah. Inventingthefuture: informationservicesforanew millenium. - Stan ford: Ablex, 1999. p. 80 . 
denominado Na tio nalDigitalLibrary Program, dela Bibliotecadel Congre sodelos Esta dos Unidos, cuyoses fuerzos es tán en ca mina dos a convertirlas colec cionesimpresasenrecursosdigitales.

Con esta vi sión la biblio te ca digital está de jan do el pa pel pa si vo de serun al ma cenamien todein formación para convertirseenunac tivodise minadordeella, ${ }^{11}$ ya que sepre ten de quela utiliza ción delos me dios tec nológicos comolas re des ylas com putadoraspermitiráelaccesoa todoelmaterialdigitalizado.Engeneralpuedeafirmarse quelabiblio te ca digital se conside racomo una opor tu nidad para conver tirlos materialesimpresosaunformatodigital.

Sin em bargo con esta con ver sión de ma te rial im pre so a digi tal no se crea una verdaderabiblioteca digital, ${ }^{12}$ tan sólo se potencializael servicio que ya da la biblioteca tradicional. El tras pa so de un forma to a otro por me dio de la digi ta liza ción permite protegerlosmate ria les an tiguos olos degran de man day facul ta que éstos pue dan ser consultados por más de un usuario a la vez. Esto constituye una alternativa para las bibliotecastradicionales, que es tán op tan do cada vez más porla di gi ta li za ción y disposiciónenreddepartedesuscolecciones.

Sin em bargo exis ten al gu nas ex cep cio nes pues en al gu nos pro yec tos de este tipo de bi blio te ca que se es tán efec tuan do en otras par tes del mun do se tie ne una vi sión más ampliadeloquedebeserunabibliotecadigital.

Porejem ploen China se es tángene ran do biblio te cas digitales en las que se pue de observarun especialén fa sis enla oferta deherra mien tasy métodos eficientes para el trabajo y el estudio a través de un modelo adecuado para usar la información, todo elloconelpropó sito deimpulsarlaedu ca ción, lainves tiga ción cien tíficay elde sarrollodetecnología. ${ }^{13}$

Otroas pec to que ca rac te riza ala casi to ta lidad delos pro yec tos de biblio te cas digitales es el ofrecimien to de catálogos públicos enlínea; ${ }^{14}$ ello de bi do a que se observalaideageneralizada de que una biblioteca digitales aquella quepermitela localización, ${ }^{15}$ de manera remota, de los documentos de una biblioteca. Por ello podemosvercon frecuen cia que se de no minabiblio te ca digital a aquella que cuentaconestetipodecatálogos.

Sin em bargo los ma te ria les que seiden ti fican a par tirde ca tá lo gos pú blicos enlí nea no necesariamente son digitales, sino que la mayor par te de las ve ces son do cumentosimpresos.Laventaja másim portantedel catálogoenlíneaessuacce soatravés

11 Ron Chepesiuk. "The future is here: America's libraries go digital”. En: American Libraries (jan. 1997). p. 47.

12 Una biblioteca digital implica también la produccióndemate riales denaturalezadigital.

13 YangZongying. "The architec tu re of digitallibrary proto tipeinShanghaiJiao Tong University".(tex to tradu cidodelchino, publica do en Informaciónsobrelaeducaciónen biblioteconomía. - Vol. 35, no. 1. - (1997). - pp. 77-92.

14 Algu nas más ya ha blan de por ta les.

15 En el sentido de iden ti fi cary de co no cer dón de se en cuen tra el ma te rial. 
dere des como In ternety, sólo enalgu nos ca sos, el ofre cimien to de aparta doenlínea deloslibros,previalarevisióndelestatusdelmaterial.

Cier toquelabiblio te ca di gitalhabráde ofrecercatálogos paralaiden tificaciónde los documentos, perose tratadedocumentosdigitalesy disponiblesen red.Entanto se tenga acceso alas bases de da tosconel re gis trode diversas biblio tecas digitales, se po drá ha blar de una co o pe ra ción en tre este tipo de bi blio te cas y a partir de ello se estableceríanserviciosdeinformación.

Frenteaeste es cenario ¿se puedehablarde servicios nuevos? En realidadés tos no pueden considerarse servicios propios de la biblioteca digital, sino servicios que le son añadidos a la biblioteca tradicional. Sin embargo la literatura en cuestión suele rei te raruna y otra vezquela biblio te ca digital presenta "un tipo de servicios de in forma ción de muy di ver so ran go que está en pro ce so de trans for ma ción”. ${ }^{16}$ ¿P ero cuálessonesosserviciosycómo serásutrans formación? Faltaex plicitar.

Mien tras tan to po de mos veral gu nos ejem plos de cómo los servicios de la bibliotecadigitalsonserviciosañadidos:

La circula ción delos ma te riales es uno de los as pec tos en los que la biblio te ca digi tal re fle ja este rasgo de po ten cia li za ción delos servi cios ya exis ten tes. La circulación que po sibilitalabiblioteca digitallareflejaclaramentecomounabiblioteca que compar te sus re cur sos, es de cir, una que no se ob ser va como un con te ne dor sino como uninterlocutor. ${ }^{17}$

Elproyecto de biblio te ca digitalque de sa rrollala Universidadde Jiao Tong, Shanghai, men cio na a la cola bo ra ción comounodelos pun tos cen trales. Sepre ten dequelos usuarios trabajencon mu chas fuen tes dein forma ciónen forma demulti-medios dis tribuidosqueprocesenadistanciayquecompartanrecursos..$^{18}$

Para lograr esta colaboración entre bibliotecas antes es necesario plantear una normalización adiferentesniveles:para elcontrol delos ma te riales digitales, su presentación, suautentificación, supreservación, etcétera. ${ }^{19}$

El caso de la circu la ción de publica cio nes elec tró ni cas y en es pe cial dellibro electrónico, obe de ce a la ten den cia co mercial que dic tan las em pre sas produc to ras, tendenciasquelabibliotecatratadereconciliarconsus prácticastradicionales. ${ }^{20}$

16 Ro salía Peña. Ges tión di gital de la in for ma ción: de bits a biblio te cas digitales yla web. - Ma drid: Rama, 2002. - p. 362.

17 José Arias O. Tallerdebibliotecasdigitales:eldesarrollodelasestructurasdelabibliotecadigital. / José Arias O.,Hernan do CruzM., Mar tha Helena Arango deV.-San ta fé,Colombia:Pon tificia Universidad Javeriana, 2000. - p. 55.

18 Yang Zongying, Op. cit., p. 77.

19 The evolving virtual library : practical and philosophical perspectives. Edited by Laverna M. Saunders. Medford, N.J : Information today, 1999.

20 Deborah Carver. "From circula tion to access services: the shiftin aca demiclibraryo rganization", en Accessservicesinlibraries:news solutionsforcollection management / Gree Sapp, edi tor. - New York: Hawoth, 1992. - p. 34. 
Sin em bargo ha cien do a un lado el pro ble ma dela ven ta delos de re chos por el uso delosdo cumen tos, que ac tual men teestá en uso, la circula ción delos libros elec tróni cosenel futu ro po drá ofrecercier tas ven tajas, ya que en elmomen to en que el usua rio solicite uno en préstamo, se generará una copia con un certificado encriptado. Tal cer ti fica do ten drála do ble fun ción de es ta ble cer el tiem po du ran te el que se le presta el libro al usua rio, y evi ta rá queéste sea en via do ha cia otrolec tor elec trónico, o que se haga una impresión de él. En caso de que el lec tor no de vuel va la co pia a tiem po, el certificadose invalidaráy automáticamente el li bro será bo rra do dellec tor delusuario.Tambiénautomáticamenteelcatálogodelabibliotecagenera ráuna nuevacopia para que ésta pue da sercon sul ta da porotros usua rios y se po drán en viarcorreos electró ni cos anun cian doque el libroestá nue va men te dis ponible parasuprés ta mo. Con elloelpréstamodelibroselectrónicostendrálas siguientescualidades:

1. No habrá retrasos en la devolución del ejemplar a la biblioteca.

2. No seráne ce sa rio en viar re cla ma cio nes y en con se cuen cia ya no exis ti ránlas mul tas para los usua rios, pero qui zá se deba pen sar en cier tas san cio nes para evi tar algún mal manejo del material en préstamo.

3. No será necesario devolver físicamente el libro, ni retirarlo de la estantería.

La ma ne ra como se in te gra rán los libros elec tró ni cos ala circula ciónen las bibliotecaspodráserdedosmaneras:

1. Lospro vee dores tra dicionales de sarrolla ránnuevos mó dulos quelepermitanala biblioteca manejar sus libros electrónicos.

2. Se crea rán nuevas orga ni za cio nes que se es pecia li za rán en el ma ne jo de estenuevo material.

3. Incluso podrán ser factibles las alian zas en trelos provee do res tra di cio nalesylas nuevas orga niza cio nes que se especialicen enla ad minis tración delibros elec trónicos.

Otraposibilidad esel prés tamodelec toreselec tró ni cos, ${ }^{21}$ en el en ten di do de que en la actualidad son caros y esto complica su adquisición por parte de los usuarios. Por estas razones en México éste sigue sien do un asun to que tie ne se rias com plicacio nes. Laideaes que es tos lec to res sean pres ta dos como cual quierotro mate rialde la bibliotecayqueelpreciodeloslibrosseacargadodeantemano.

Para elprés tamointerbibliotecarioseusaránpáginaswebdonde secargueelprecio porloslibros. Me dian te una cla ve es pe cial, las biblio te cas in te resa das en de terminado mate rial po drán aho rrar tiem po y dine ropara ob tener títulos que de otra ma ne rase ría complicadoconsultar. Esto nos podría es taracercan do a un plan de coo pera ción que funcioneconmayoreficienciaybeneficiosparalas bibliotecasysususuarios.

En el aspecto de la selección decolec cionesloslibroselec tró nicosofrecen va rias ven tajas, como el aho rro del tiem po en el que se pone a dis po si ción del usua rio el materialqueleinteresa. Otraventajaesquenosenece si ta rámu choespacio paraguardar

21 Dispositivos que se requieren para la lectura de este tipo de publicaciones. 
es tos li bros en la bi blio te ca y esto con tribui ría a la re duc ción de cos tos parain ven tario, descarte,restauraciónyorganizaciónenlaestanteríadeloslibros.

Ademásellibroelec tró nico permiti rábús que das máságiles, ya queal teneracceso al texto completo el usuario tendrá mayor seguridad acerca de la información que le ofrece el libro. En este sentido, la efi cien cia de los servi cios se gui rá de pendiendodela efectividad que ofrezca el regis trodelos do cumentos digitales, sinperderde vis ta quela sis te ma tiza ción de es tos do cu men tos de be rá per mi tirex ten der los servi cios tra di cio na les y no cons ti tuir se en un conjunto ais la do de la cons tela cióndeimpresos. 22

Sicomo ya se se ñaló, la biblio te ca digitalno tie ne como ca rac te rís ticaúni ca el ser contene dorade to doslos documentos digitales quelecompeten, sinotambiéncompartir su colección con otras bibliotecas, ${ }^{23}$ estaríamos hablando de la necesidad de crearcatálogoscolectivosenlínea.

Precisamente a partir de la agilidad que se ofrezca en la búsqueda y el acceso a los mate riales bibliotecarios sepodrán crearservicios comoeldecon sulta, endon de see speraqueimpereloquehallega doalla marse autoservicio; esa forma directadebúsqueday recuperacióndelainformación, sinintermediacióndelbibliotecariodeconsulta. ${ }^{24}$

En la ac tua lidad se dis cu te mu cho cómo po dría ser el servicio de con sul ta en labibliote ca di gital y con fre cuen cia selle van a cabo even tos de dica dos al análi sis de este aspecto, ${ }^{25}$ pues todavía no setienen muyclaraslasventajasquetraeráconsigo.

In clu so se con side ra quelas ca rac te rís ticas de este servicio per mea rán a to doslos demás que se ofrezcan a tra vés de la biblio te ca digital. Sise con sidera que una biblioteca digital debe contar con texto completo, es fácil saber de antemano que la búsque da y re cu pe ra ción de la in for ma ción la hará el usua rio de ma ne ra di rec ta, no sólo medianteloquetodavíadenominamosserviciodeconsulta.

Ental es ce na rio elbiblio te cario ten dríala opor tu ni dad de ofre cerservicios como el de diseminación selectiva de la información de manera más rápida y confiable, puesto que las tecnologías le permiti rían una mejor aproxima ción ala recuperación del material pertinente para cada usuario. La biblioteca digital no será un elemento que propiciela to tal ex clu sión del biblio te ca rio, sino quele ayu da rá a ahorrar tiem po yes fuerzo,asícomoaincrementarsuefectividadeneldesarrollodesuactividad.

22 Da niel Grens tein. The digital library : a biography. - Washington, D.C : Digital Library Federation, Coun cil on Li brary and In for ma tion Re sour ces, 2002. - p. 25.

23 Colec ción que se con for ma ría, casi en su ma yo ría, al in te rior de la ins ti tu ción de lacual de pen da la biblio te ca. Eneste sentido, imperalaidea de que la biblio te ca digital ten dría un ma yordesa rrolloen elámbitoacadémico, dondeprofesoreseinves tigadoresproduciríntex tos decali dad que bien podrían formar parte de labiblio teca.

24 Toni Carbo. "Toward the fourth era of information services : just for you services", en NFAIS newsletter. - Vol. 40, no. 3. - (1998). - p. 36.

25 Para conocer algunas de estos eventos se puede visitar el sitio": http//www.vrd.org/conf-train.shtml 
Lasanteriorescaracterísticas revelan una me jo ría en los servi cios de con sul ta, de dise mina cióny de prés ta mo que des de hace tiempose ofre cea tra vés delabiblioteca tradicional.

Haciendo una analogía entre los servicios de la biblioteca tradicional y la digital puedeobservarselosiguiente:

\begin{tabular}{|c|c|}
\hline Biblioteca tradicional & Biblioteca digital \\
\hline & $\begin{array}{l}\text { Sus al can ces y límites se en cuen tran de li mi ta dos por } \\
\text { eldesarrollotecnológico. }\end{array}$ \\
\hline $\begin{array}{l}\text { Su mo de lo de servicios se des pren de } \\
\text { delos procesos editoriales. }\end{array}$ & $\begin{array}{l}\text { Su mo de lo de ser vi cios de pen de del mo de lo de pur } \\
\text { blicacióndigital. }\end{array}$ \\
\hline $\begin{array}{l}\text { La pre ser va ción es lo más im por tan- } \\
\text { te para la bi blio te ca }\end{array}$ & $\begin{array}{l}\text { Se im pul sa la crea ción de co lec cio nes a par tir de la } \\
\text { digitalización de impresos. } \\
\text { Adquiereunpredominantepapelde disemina dor. }\end{array}$ \\
\hline $\begin{array}{l}\text { Se in ten tan crear reper to rios univer- } \\
\text { sales. }\end{array}$ & $\begin{array}{l}\text { Se crean ca tá lo gos pú bli cos en línea, ten dien tes a } \\
\text { iden tificareluniversodepublicaciones. }\end{array}$ \\
\hline $\begin{array}{l}\text { Se tie ne el sue ño de crear gran des bi- } \\
\text { blio te cas que al ber guen eluniverso } \\
\text { depublicaciones. }\end{array}$ & $\begin{array}{l}\text { Aunque pre ten dela uni ver salidad, sólo con tem pla } \\
\text { documentosdigitales. }\end{array}$ \\
\hline $\begin{array}{l}\text { Se da la in terme diación en la oferta } \\
\text { deservicios. }\end{array}$ & Surge el concep to de "au to servicio". \\
\hline Prevaleceelserviciopúblico. & $\begin{array}{l}\text { El servi cio pú bli co se di lu ye. Pre va le ce el co bro para } \\
\text { elacceso. }\end{array}$ \\
\hline $\begin{array}{l}\text { Ofrece servicios biblio te ca rios y al- } \\
\text { gunos servicios dein formación. }\end{array}$ & Ofreceservicios dein formación. \\
\hline $\begin{array}{l}\text { Los servi cios de prés ta mo y con sul ta } \\
\text { son los más im por tan tes. }\end{array}$ & $\begin{array}{l}\text { Desaparece el concep to deprés ta mo. Prevale ceyse } \\
\text { for taleceel serviciodecon sul ta. }\end{array}$ \\
\hline $\begin{array}{l}\text { Se ofrecen servicios de dise mina ción } \\
\text { selectiva y entregadedocumentos. }\end{array}$ & $\begin{array}{l}\text { Se da el ser vi cio de en tre ga de do cu men tos vía In ter- } \\
\text { net y se per so na li zan cada vez más los ser vi cios. }\end{array}$ \\
\hline \multirow[t]{2}{*}{$\begin{array}{l}\text { Su ti po lo gía es más am plia que la } \\
\text { quepermi te la biblio te ca digital. }\end{array}$} & $\begin{array}{l}\text { Por sus ca rac te rís ti cas se tien de a la crea ción de bi- } \\
\text { bliotecas digitales de carácter académico y a la cons- } \\
\text { titu ción deredes. }\end{array}$ \\
\hline & $\begin{array}{l}\text { Sus ser vi cios com ple men tan y/o po ten cian los tra di- } \\
\text { cionales. }\end{array}$ \\
\hline
\end{tabular}

De acuerdo con lo visto se pueden establecer diagramas comparativos entre los servicios de la biblioteca tradicional y de la digital, que permiten apreciar mejor sus elementos: 


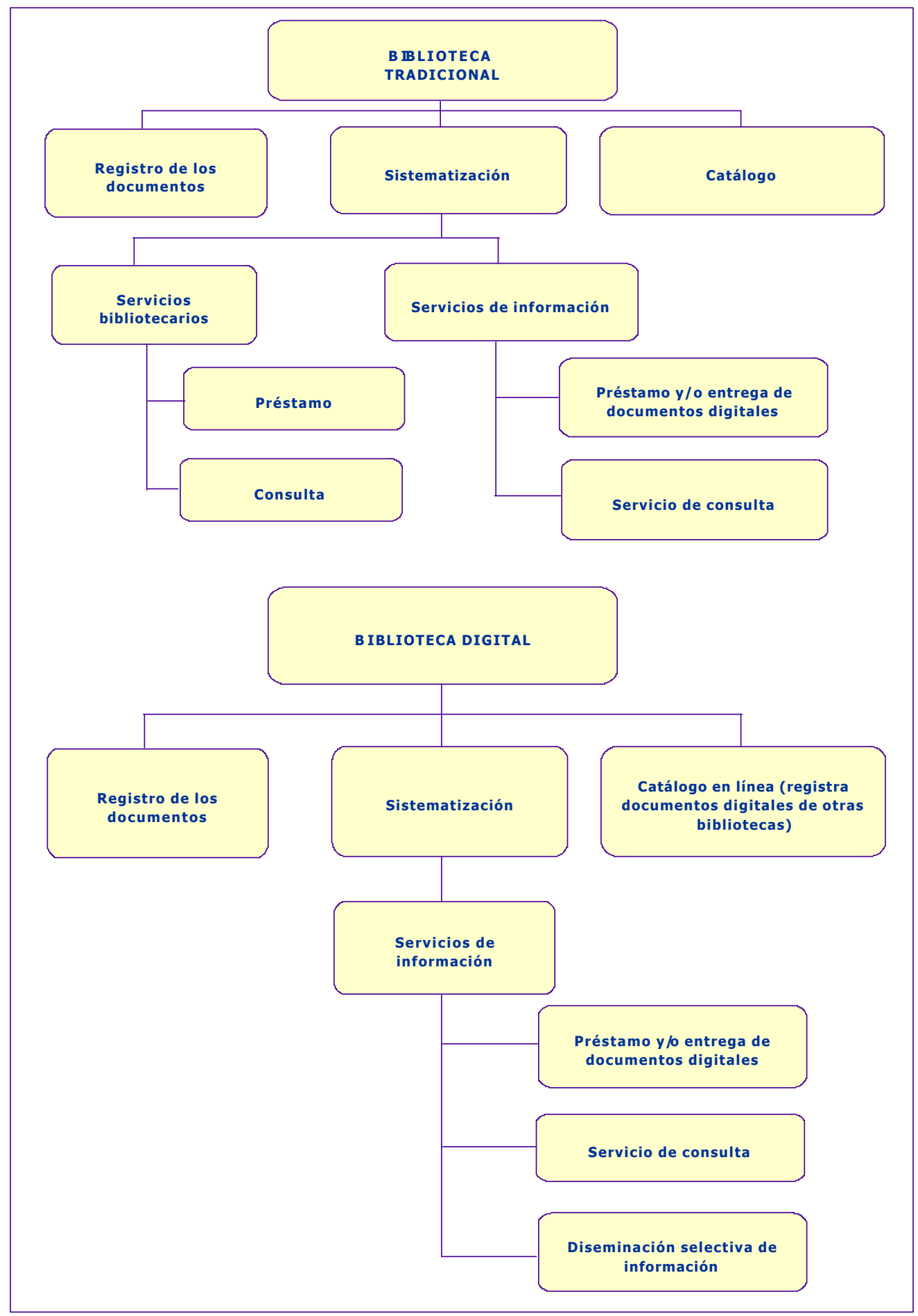




\section{CONSIDERACIONES FINALES}

Conbaseenloanterior, sepue de concluirque:

* Los servicios delabiblio te ca digitalse de benabordardes dela pers pec tiva de los servicios de información, en tanto que se basan en la cooperación a través de redes.

* Elpoten cialylímites de es tos servi cios se en cuen tran de li nea dos porlotecnológico.

* La biblioteca digital ofrece servicios adicionales que potencian los ya existentes en la biblioteca convencional. En este sentido no puede hablarse de nue vos ser vi cios sino de los mis mos, pero bajo pro ce sos que per mi ten mayor amplitud.

* La gama de servicios que ofrece la biblioteca digital se pueden agrupar en:

$>$ servicios de consulta y

$>$ Diseminación Selectiva de la Información (DSI)

Es tosservicios depen de rán delain fraestruc turatec nológicadeque se disponga, pero tam bién dela orga niza ción que sehaga delos do cu men tos, asícomo de las polí ticas y as pec toslega les que se es ta blez can para su uso (au ten ticidad delos documentos, confidencialidad, autenticidaddelusuario,pagos, etcétera).Quizáunavezque se ven zan es tos obs táculos po dremos ha blar de nuevos servicios, porlo pron to son tan sóloquimeras.

\section{BIBLIOGRAFÍA}

Achinstein, Peter. Los modelosteóricos. - UNAM, Direc ción Ge ne ral de Put blicaciones, 1967.

Chepesiuk, Ron. “The future is here: America's libraries go digital”. En: American Libraries (jan 1997).

Arias O., José. Tallerdebibliote cas digitales: elde sa rro llo de las estructuras de la bi bliotecadigital. / José Arias O., Her nan do Cruz M., Mar tha Hele na ArangodeV.-Santafé,Colombia:PontificiaUniversidadJaveriana,2000.

Carbo, Toni. Toward the fourth era of in for ma tion ser vi ces : just for you services, en NFAIS newsletter. - Vol. 40, no. 3. - (1998).

Carver, De bo rah. From cir cu la tion to ac cess services : the shift in aca demiclibraryorganization, en Access services in libraries:new solutionsforcollec tion management / Gree Sapp, editor. - New York: Hawoth, 1992.

Theevolvingvirtuallibrary:practicalandphilosophicalperspectives / Edi ted by Laverna M. Saunders. - Medford, N.J : Information today, 1999.

Foskett, D.J. Information service in libraries. - India : Akashdeep, 1992.

GarcíaCamarero,Ernesto. Labibliotecadigital/Ernes to GarcíaCama rero, Luis Angel García Melero.- Madrid : Arcos, 2001. 
Grens tein, Da niel. The digitallibrary: a biography. - Wa shing ton, D.C:Di gi talLibraryFederation, CouncilonLibraryand In formation Resources, 2002.

Hannah, Stan A. Inven ting thefuture:information servicesfor anew mille nium. Stanford: Ablex, 1999.

Hurley, BernardJ. Themaking of AmericaIItestbedproject: a digitallibrary servi ce model. - Washington, D.C. :The Digital Library Federation, 2000.

Martin, Susan K. Library networks, 1986-87: libraries in partnership. - New York : Knowledge Industry Publications, 1986.

Peña, Ro salía. Ges tión digital dela in for ma ción: de bits a biblio te cas digi tales y la web. - Madrid : Rama, 2002.

Torres Vargas, Georgina Araceli. La bibliotecavirtual ¿qué es y qué promete? : UNAM, CUIB, 2000.

Torres Vargas, Georgina Ara celi. “Los servi cios biblio te ca rios y de in formaciónen elcon tex to delabiblio te ca tra dicional”, en Investigaciónbibliotecológica. - Vol. 15, no. 31. - (jul.-dic., 2001).

Zongying, Yang. "The architec tu re of digitallibraryprototipein Shanghai Jiao Tong University". - (tex to tra du cido del chi no, publica do en Información sobre la educación en biblioteconomía. - Vol. 35, no. 1. - (1997). 